\title{
The impact of several craniotomies on transcranial motor evoked potential monitoring during neurosurgery
}

\author{
Ryosuke Tomio, MD, Takenori Akiyama, MD, PhD, Masahiro Toda, MD, PhD, \\ Takayuki Ohira, MD, PhD, and Kazunari Yoshida, MD, PhD \\ Department of Neurosurgery, School of Medicine, Keio University, Tokyo, Japan
}

\begin{abstract}
OBJECTIVE Transcranial motor evoked potential (tMEP) monitoring is popular in neurosurgery; however, the accuracy of tMEP can be impaired by craniotomy. Each craniotomy procedure and changes in the CSF levels affects the current spread. The aim of this study was to investigate the influence of several craniotomies on tMEP monitoring by using C3-4 transcranial electrical stimulation (TES).

METHODS The authors used the finite element method to visualize the electric field in the brain, which was generated by TES, using realistic 3D head models developed from T1-weighted MR images. Surfaces of 5 layers of the head (brain, CSF, skull, subcutaneous fat, and skin layer) were separated as accurately as possible. The authors created 5 models of the head, as follows: normal head; frontotemporal craniotomy; parietal craniotomy; temporal craniotomy; and occipital craniotomy. The computer simulation was investigated by finite element methods, and clinical recordings of the stimulation threshold level of upper-extremity tMEP (UE-tMEP) during neurosurgery were also studied in 30 patients to validate the simulation study.
\end{abstract}

RESULTS Bone removal during the craniotomy positively affected the generation of the electric field in the motor cortex if the motor cortex was just under the bone at the margin of the craniotomy window. This finding from the authors' simulation study was consistent with clinical reports of frontotemporal craniotomy cases. A major decrease in CSF levels during an operation had a significantly negative impact on the electric field when the motor cortex was exposed to air. The CSF surface level during neurosurgery depends on the body position and location of the craniotomy. The parietal craniotomy and temporal craniotomy were susceptible to the effect of the changing CSF level, based on the simulation study. A marked increase in the threshold following a decrease in CSF was actually recorded in clinical reports of the UE-tMEP threshold from a temporal craniotomy. However, most frontotemporal craniotomy cases were minimally affected by a small decrease in CSF.

CONCLUSIONS Bone removal during a craniotomy positively affects the generation of the electric field in the motor cortex if the motor cortex is just under the bone at the margin of the craniotomy window. The CSF decrease and the shifting brain can negatively affect tMEP ignition. These changes should be minimized to maintain the original conductivity between the motor cortex and the skull, and the operation team must remember the fluctuation of the tMEP threshold. https://thejns.org/doi/abs/10.3171/2016.7.JNS152759

KEY WORDS transcranial motor evoked potential; transcranial electrical stimulation; finite element method; craniotomy; neurosurgery; diagnostic technique

$\mathrm{T}$ HE intraoperative monitoring of motor evoked potentials (MEPs) by transcranial electrical stimulation (TES) is popular for motor function preservation. ${ }^{6-8,11}$ Transcranial MEP (tMEP) monitoring is more readily performed than cortical MEP (cMEP); however, tMEP is considered less accurate than cMEP, ${ }^{2,4,9}$ Shifts in the tMEP stimulation threshold during craniotomy are well known. In our limited experience, the threshold decreases slightly after the craniotomy if the anesthetic depth and other conditions are equal, but increases during long surgical procedures. The craniotomy procedure and changes in the CSF levels would affect the current spread during TES. These changes can impair the accuracy of tMEP monitoring.

ABBREVIATIONS AC = alternating current; $A V M=$ arteriovenous malformation; $C M E P$, tMEP, UE-tMEP = cortical motor evoked potential, transcranial MEP, upper-extremity tMEP; $D C=$ direct current; $F E=$ finite element; $L S O=$ lateral suboccipital; $p E F, t E F=$ perpendicular electric field, tangential $E F ; T E S=$ transcranial electrical stimulation. SUBMITTED November 26, 2015. ACCEPTED July 13, 2016. 
The electric field produced by TES in the human brain cannot be investigated using in vivo or in vitro studies. Therefore, we calculated the electric field in the brain during tMEP monitoring by using realistic finite element (FE) models generated from standard brain MR images. Some authors have reported the usefulness of this method for evaluating the electric field in the brain during therapeutic transcranial direct current (DC) stimulation. ${ }^{1,3}$ We previously reported the visualization of the electric field produced by TES during frontotemporal craniotomy by using realistic 3D head models and the FE method. ${ }^{10}$ However, to our knowledge, no report in the literature compares the electric field for tMEP monitoring during several craniotomies and body positions.

The aim of this study was to investigate the influence of the craniotomy procedures and positions on tMEP monitoring during neurosurgery. We investigated the computer simulation by FE methods, and we also studied clinical recordings of the stimulation threshold level of upperextremity tMEP (UE-tMEP) during neurosurgery in 30 patients to validate the simulation study.

\section{Methods}

\section{Model Simulation Study}

Realistic 3D Head Model Creation

The realistic 3D head models created in this study were developed from International Consortium for Brain Mapping (ICBM) T1-weighted MR images obtained from BrainWeb (http://brainweb.bic.mni.mcgill.ca/). The image processing and segmentation of $1 \times 1 \times 1 \mathrm{~mm}^{3}$-resolution images were performed using ScanIP and + ScanFE (version 6.0, Simpleware Ltd.). The images of the brain, CSF, skull, subcutaneous fat, and skin layer were obtained from the T1-weighted MRI studies. These FE models were meshed into more than $1.5 \times 10^{7}$ tetrahedral elements for the $1 \times 1 \times 1 \mathrm{~mm}^{3}$-resolution supratentorial brain models.

Next, we created 4 models of craniotomy-frontotemporal craniotomy, parietal craniotomy, temporal craniotomy, and occipital craniotomy - by using the 3D processing tool in ScanIP (Fig. 1). We simulated each craniotomy model as is usually done in neurosurgery. The skin layer and bone layer were removed in the craniotomy models. The CSF-decreased models were designed by removing the CSF layer to simulate the intraoperative situation of decreased CSF surface level with each body position.

\section{Electrode Placement}

Corkscrew-type bipolar electrodes were placed on the scalp at sites C3 and C4 (based on the international 10-20 system); the anode was placed at $\mathrm{C} 3$ and the cathode was placed at $\mathrm{C} 4$. The corkscrew-type electrode was created by computer-aided design to imitate a popular design. The details of the electrode design and the manner of placement were the same as in our previous publication. ${ }^{10}$

\section{Tissue Conduction Properties and Calculations by Comsol Multiphysics}

All tissue layers were modeled as homogeneous and isotropic with respect to electrical conductivity and permittivity based on data from the human organs property database for computing simulation (http://cfd-duo.riken. go.jp/cbms-mplindex.htm). The details of the conductivity values were the same as in our previous publication, ${ }^{10}$ and its values were not the same but were nearly consistent with prior reports. ${ }^{1,5}$ The FE mesh exported by ScanIP was read into Comsol Multiphysics (version 5.1, Comsol AB). The electric field was calculated statically using the constant DC application mode of the alternating current (AC)/DC module in Comsol, which solved Laplace's equation for the electric potential. The boundary conditions assumed the following: 1) uniform inward current flow applied to the distal surface of the anode electrode; 2) ground applied to the distal surface of the cathode electrode; 3 ) injected current set to $100 \mathrm{~mA}$, because $100 \mathrm{~mA}$ usually induces both the upper- and lower-extremity MEPs simultaneously; and 4) all other external boundaries were insulated. The calculation by Comsol Multiphysics took less than 120 minutes for the $1 \times 1 \times 1 \mathrm{~mm}^{3}$-resolution models, using a Corei7-4710 CPU and 32 GB of RAM.

\section{Analysis of the Results}

We studied the electric field plotted on the cross-sections and brain surface in each model. A coronal section that contained both the electrodes was made, and axial sections were cut at the center of the stimulated cortex. The magnitude of the electric field was estimated in all models. Both the magnitude of the component of the electric field tangential (tEF) to the brain interface and perpendicular ( $\mathrm{pEF}$ ) to the brain interface were estimated in the surface studies. Although the perpendicular component is negative where the field is inward to the surface and positive where the field is outward to the surface, we plotted the absolute values of the perpendicular component in our surface studies. The electric field values of all cross-section studies were visualized by a color scale ranging from $0 \mathrm{~V} / \mathrm{m}$ (blue) to $60 \mathrm{~V} / \mathrm{m}$ (red).

\section{Limitations of the Models}

The main technical limitations of the FE method simulation were the same as in our previous report. The limitation details regarding the use of an isotropic layer, the DC application mode by Comsol Multiphysics, and the mesh generation are described in our previous publication. ${ }^{10}$

Our "CSF-decreased" models seemed to be overly simple because we simplified the subarachnoid space into a single reservoir. All the compartments of the subarachnoid space should be intricately connected at the micro level, but the subarachnoid space is divided at the macro level and its communication is partially restricted. Therefore, our models may not represent the actual pattern of CSF decrease, and this is the most important limitation of this study. Nevertheless, our models show an effect of a major decrease in CSF in simple simulations.

\section{Clinical Validation With Recording of the tMEP Thresholds Patient Population}

Intraoperative tMEP monitoring and stimulation threshold recording were applied to a total of 30 patients consisting of 9 men and 21 women ranging in age from 19 to 74 years (mean 55.7 years). All patients underwent a crani- 

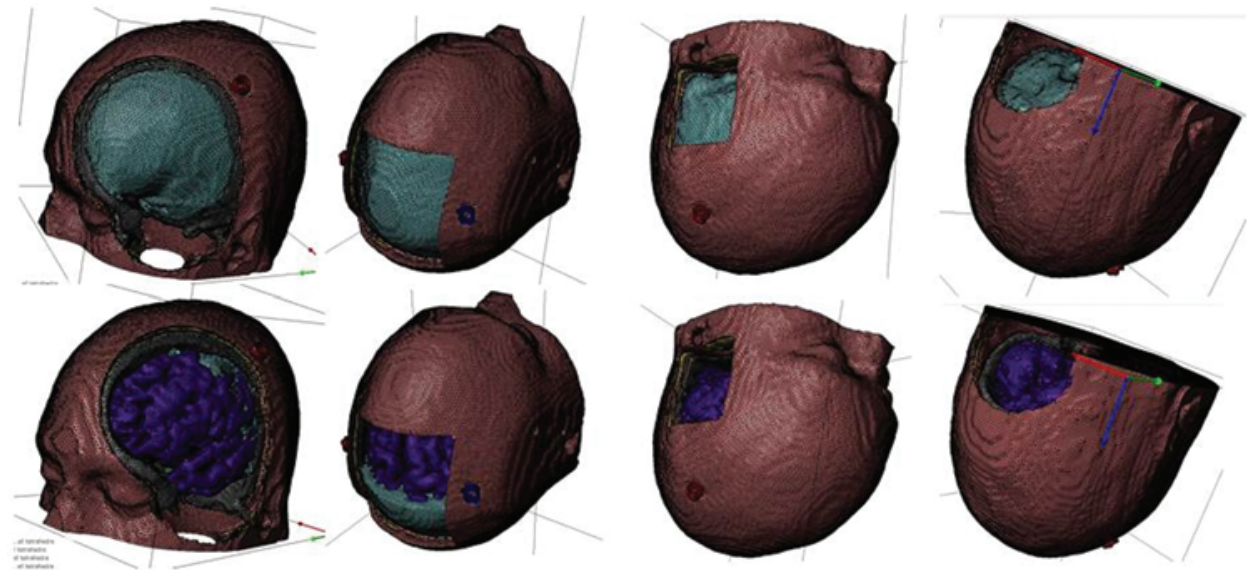

FIG. 1. The craniotomy models (upper row) and CSF-decreased models (lower row) in $1 \times 1 \times 1 \mathrm{~mm}^{3}$-resolution images. Starting from the left, the frontotemporal craniotomy model, parietal craniotomy model, temporal craniotomy model, and occipital craniotomy model are presented. Figure is available in color online only.

otomy. There were 13 frontotemporal craniotomy cases, 3 bifrontal craniotomy cases, 2 parietal craniotomy cases, 5 temporal craniotomy cases, 1 occipital craniotomy case, and 6 lateral suboccipital (LSO) craniotomy cases. Patients with motor weakness before surgery were excluded.

Clinical data recording for this study was in accordance with the ethical standards of the institutional review board of Keio University and with the Helsinki Declaration of 1975, as revised in 2000 and 2008. Informed consent for neurophysiological monitoring was also obtained before surgery.

\section{Neurophysiological Monitoring Methods and Recording Settings}

The corkscrew electrode placements at $\mathrm{C} 3$ and $\mathrm{C} 4$ were based on the international 10-20 system. We recorded tMEP thresholds during neurosurgery. The first recording was performed after induction of general anesthesia but before incision of the skin. The second recording was performed after bone flap removal but before dural incision. The third recording was performed after dural incision and CSF drainage. We used an MEE-1200 series intraoperative monitoring system (Nihon Kohden Co. Ltd.) as the electrophysiological device. Anodal electrical constant current stimulation was performed with short trains of 5 stimuli consisting of rectangular pulses, with a pulse duration of $0.5 \mathrm{msec}$ and an interstimulus interval of $2 \mathrm{msec}$. Compound muscle action potentials of the abductor pollicis brevis were recorded as UE-tMEPs. A bandpass filter was set from 30 to $3000 \mathrm{~Hz}$. The stimulation threshold was judged as a level of stimulation current (in $\mathrm{mA}$ ) that constantly elicits compound muscle action potentials of greater than $50 \mu \mathrm{V}$. The $\mathrm{C} 3$-anodal and $\mathrm{C} 4$-anodal stimulation recordings were sequentially performed. The data from each side were categorized as the craniotomy side or the unaffected side during the analysis of the frontotemporal, temporal, and LSO craniotomy cases. Occipital, parietal, and bifrontal craniotomy case data were categorized as the right side or the left side. We calculated the percent change in threshold values between 1) the first recordings and postcraniotomy recordings; 2) the first recordings and CSF-decreased recordings; and
3) postcraniotomy recordings and the CSF-decreased recordings.

Threshold levels are affected by the depth of anesthesia and other systemic conditions such as body temperature, blood pressure, and heart rate. Although anesthesiologists generally attempt to keep these conditions steady during neurosurgery, some change is unavoidable. Thus, we focused on the change in threshold on the unaffected side during unilateral craniotomies, such as frontotemporal and lateral craniotomies. Because this side is not affected by the craniotomy, its threshold change probably reflects the depth of the anesthesia or other systemic conditions. Thus it can be used to control for threshold changes observed on the craniotomy side. Analysis of the data by ttest was performed with SPSS Statistics 23 (IBM Corp.). The percent changes between threshold recordings are expressed as the average \pm SD.

\section{Anesthesia}

Anesthesia was induced with a bolus of propofol and remifentanil, and maintained with propofol and remifentanil at an average dosage. A short-acting muscle relaxant was induced as a bolus for intubation purposes. Although we did not use the "train of 4 stimuli" technique to test the level of relaxation due to time constraints prior to surgery, we routinely used neostigmine as a reversal agent for the muscle relaxant after intubation and before the recording of the tMEP threshold.

\section{Results}

\section{Model Simulation Study}

The electric field in the brain radially diffused from the brain surface at a maximum just below the electrodes in all of the coronal and axial sections of our simplified normal head model (Fig. 2). The electric field of the brain surface, and the magnitude of the tEF and $\mathrm{pEF}$ components also were shown. The extent of the $\mathrm{pEF}$ was narrower than that of the tEF.

The craniotomy models were compared with the estimate of the effect of each craniotomy and body position 

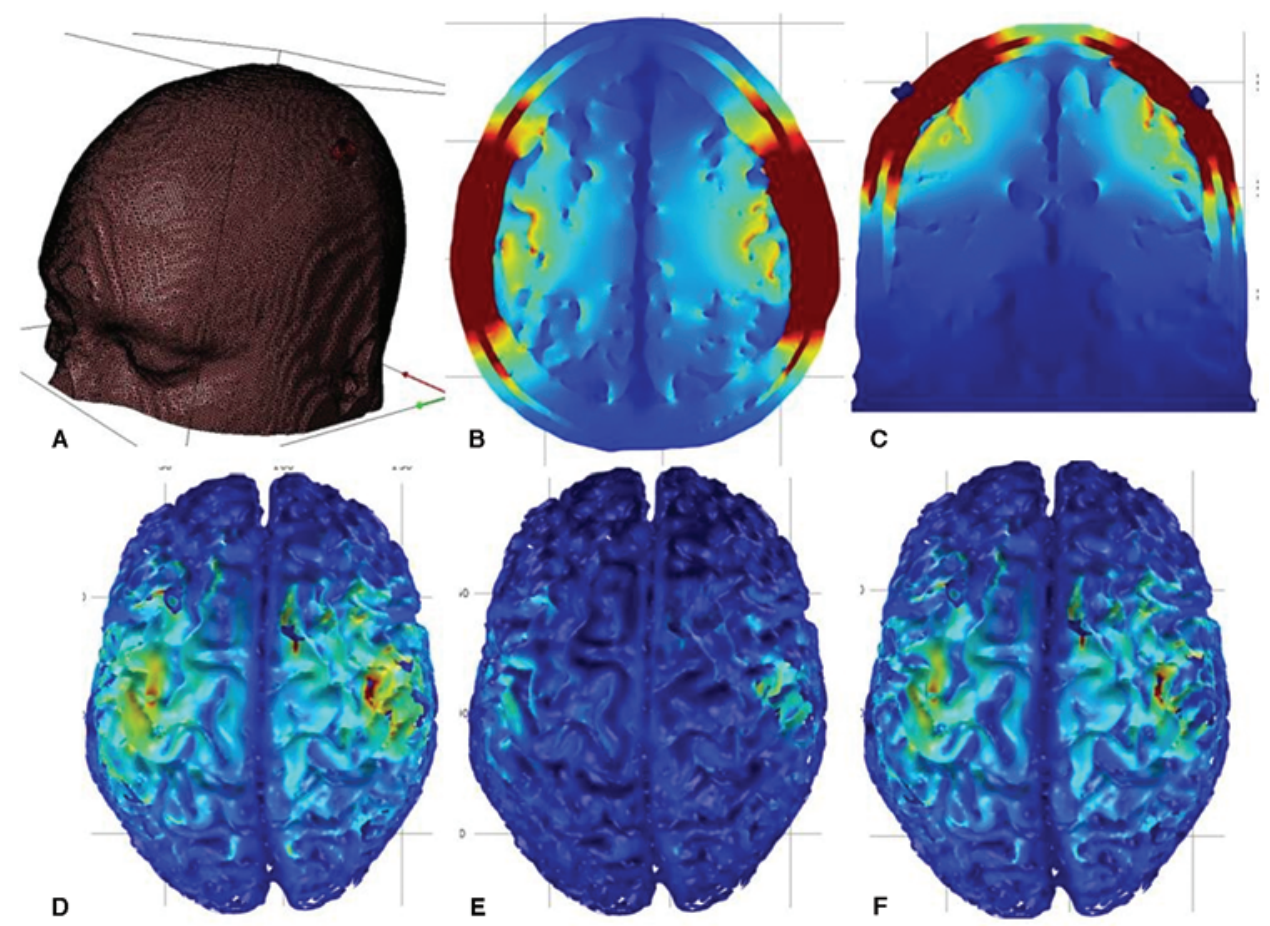

FIG. 2. The electric field of the normal head models is shown (A). The electric field in the brain radially diffuses from the brain surface at a maximum just below the electrodes in the axial and coronal sections (B and $\mathbf{C}$ ). The electric field of the brain surface (D) and the pEF component (E) and tEF component (F) of the normal head model are also shown. Figure is available in color online only.

(Fig. 3). The CSF layer was preserved in these craniotomy models. The electric field in the brain under the craniotomy window was weaker than that of the normal head model in the frontotemporal, parietal, and temporal craniotomy models. However, the removal of the bone flap increased the electric field in the brain just under the bone at the margin of the craniotomy window. The bone removal leads to a slight concentration of the electric field at the margin in these models. The electric field in the motor cortex of the craniotomy side slightly increased in the frontotemporal and temporal craniotomy models. The occipital craniotomy model was not influenced by craniotomy, because the craniotomy window was far from the stimulation site in this type of craniotomy.

Figure 4 shows that a large decrease in the CSF level has a major impact on the electric field distribution in all craniotomy models. The absence of the electric field was observed on the brain surface area above the CSF surface. The electric field on the localized brain surface just under the CSF surface was higher. A shift in the electric field distribution to under the CSF surface was also observed in these models. These findings indicate that the decrease in the CSF volume caused an uneven distribution in the electric field under the CSF surface. Impact on the motor cortex was large, especially in the frontotemporal, parietal, and temporal craniotomy models. The impact of the CSF decrease on the motor cortex depends on the body position and location of the craniotomy. Sporadically high electric fields emerging from the CSF surface were identified on the brain surface area in the CSF-decreased models. These extremely high electric field points matched closely with points in which the brain directly contacted the skull in the model. This result indicates that an extremely high electric field is generated in the brain when the brain is in direct contact with the skull during the CSF decrease. The electric field distribution in the motor cortex was not influenced as much by the CSF decrease in the occipital craniotomy model.

\section{Clinical Validation by tMEP Threshold Recordings Frontotemporal Craniotomy}

We recorded 13 frontotemporal craniotomy cases. The head was rotated $30^{\circ}-45^{\circ}$ and the vertex was tilted slightly downward in a supine position. The average percent change of the UE-tMEP threshold after craniotomy was $-6.76 \% \pm 18.1 \%$ on the craniotomy side, and that was larger than the $-0.348 \% \pm 6.55 \%$ change on the unaffected side (independent sample t-test, $p=0.019)$ (Table 1). After the CSF decrease (the 3rd recording), the average percent change of the UE-tMEP threshold from craniotomy (the 2nd recording) was $-1.73 \% \pm 11.9 \%$ on the craniotomy side and $-0.637 \% \pm 6.46 \%$ on the unaffected side $(\mathrm{p}=$ 0.051). And the percent change between the first recording and after the CSF decrease (the 3rd recording) was $-9.29 \% \pm 15.9 \%$ on the craniotomy side and $-0.88 \% \pm$ $8.65 \%$ on the unaffected side $(\mathrm{p}=0.07)$.

The percent change after the craniotomy was significantly larger on the craniotomy side than on the unaffected side. Many patients demonstrated a decreased threshold after the craniotomy, which minimally changed after the CSF decrease. The patient in Case 8, who underwent re- 


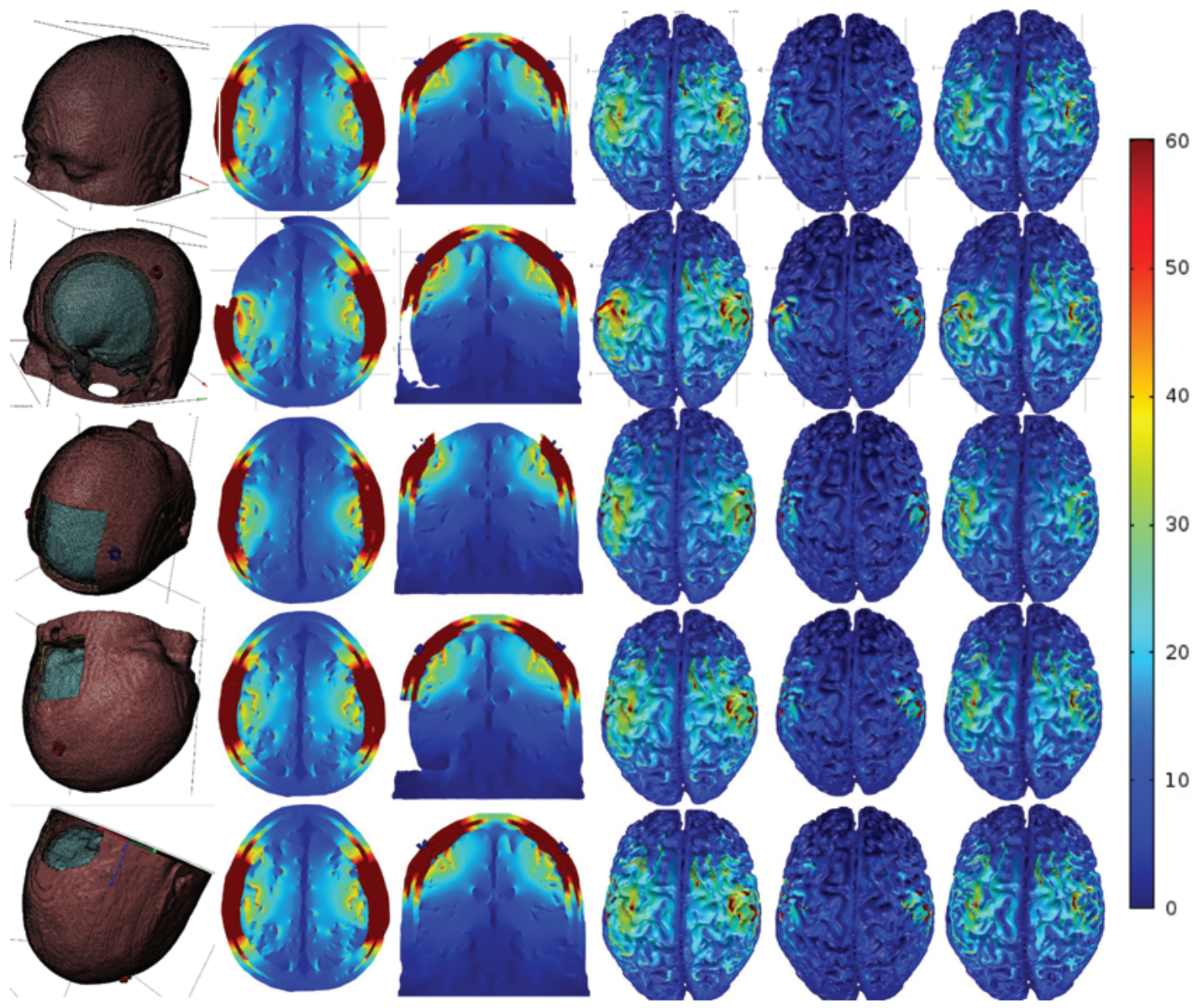

FIG. 3. The electric field in each craniotomy model is shown (from the top: normal head, frontotemporal craniotomy, parietal craniotomy, temporal craniotomy, and occipital craniotomy). Starting from the left, the 3D model view, axial section, coronal section, surface view of the electric field, $\mathrm{pEF}$ component, and $\mathrm{tEF}$ component are shown for each model. The color scale ranges from 0 $\mathrm{V} / \mathrm{m}$ (b/ue) to $60 \mathrm{~V} / \mathrm{m}(\mathrm{red})$. Figure is available in color online only.

moval of a small tuberculum sellae meningioma via right frontotemporal craniotomy, showed a marked decrease in the threshold after the craniotomy only on the craniotomy side (from $35 \mathrm{~mA}$ to $28 \mathrm{~mA},-20 \%$ ) (Fig. 5A). The patient in Case 10, who underwent convexity meningioma removal, showed a threshold decrease on the craniotomy side (from $49 \mathrm{~mA}$ to $30 \mathrm{~mA},-38.8 \%$ ) (Fig. 5B). The patient in Case 11, who underwent internal carotid artery aneurysm clipping, also demonstrated a threshold decrease on the craniotomy side (from $28 \mathrm{~mA}$ to $23 \mathrm{~mA},-17.8 \%$ ), followed by a slight increase after the CSF decrease (from $23 \mathrm{~mA}$ to $25 \mathrm{~mA}, 8.7 \%$ ) (Fig. 5C).

\section{Parietal and Bifrontal Craniotomy}

Unfortunately, the parietal craniotomy cases were not treated with patients in the supine position that resembled our 3D model. The patient in Case 1, who underwent removal of multiple meningiomas via parietal craniotomy, was in the prone position. However, the UE-tMEP threshold increased after skin flap inversion (from $25 \mathrm{~mA}$ to 42 $\mathrm{mA}, 68 \%$ ) because the electrode was detached from the skull along with the inverted skin flap (Fig. 6A). The patient in Case 2, who also underwent parietooccipital craniotomy in the prone position, had a decreased threshold after the craniotomy (from $62 \mathrm{~mA}$ to $46 \mathrm{~mA},-25.8 \%$ ), and the threshold further decreased following the CSF decrease (from $46 \mathrm{~mA}$ to $34 \mathrm{~mA},-26.1 \%$ ) (Fig. 6B). The motor cortex in this case was not exposed, but it appeared to be located just below the craniotomy window and CSF surface. The brain sank after incision of the dura mater, and the distance between the skull and the motor cortex was probably shortened due to this patient's head position. A situation like this would positively affect MEP ignition in this case.

We encountered 3 cases of bifrontal craniotomy performed with patients in the supine position, and the threshold increased in 2 of these 3 cases. The threshold values were minimally changed after the CSF decrease in 2 of these 3 cases. The craniotomy window and the CSF surface were located further anterior than the motor cortex in all 3 cases. Thus, the influence of the craniotomy seemed to be small in these bifrontal craniotomies. One of the bifrontal craniotomy cases is shown in Fig. 6C.

\section{Temporal Craniotomy}

We recorded 5 temporal craniotomy cases. The head was rotated $90^{\circ}$ with the patient in the supine position. The average percent change in the UE-tMEP threshold after the 


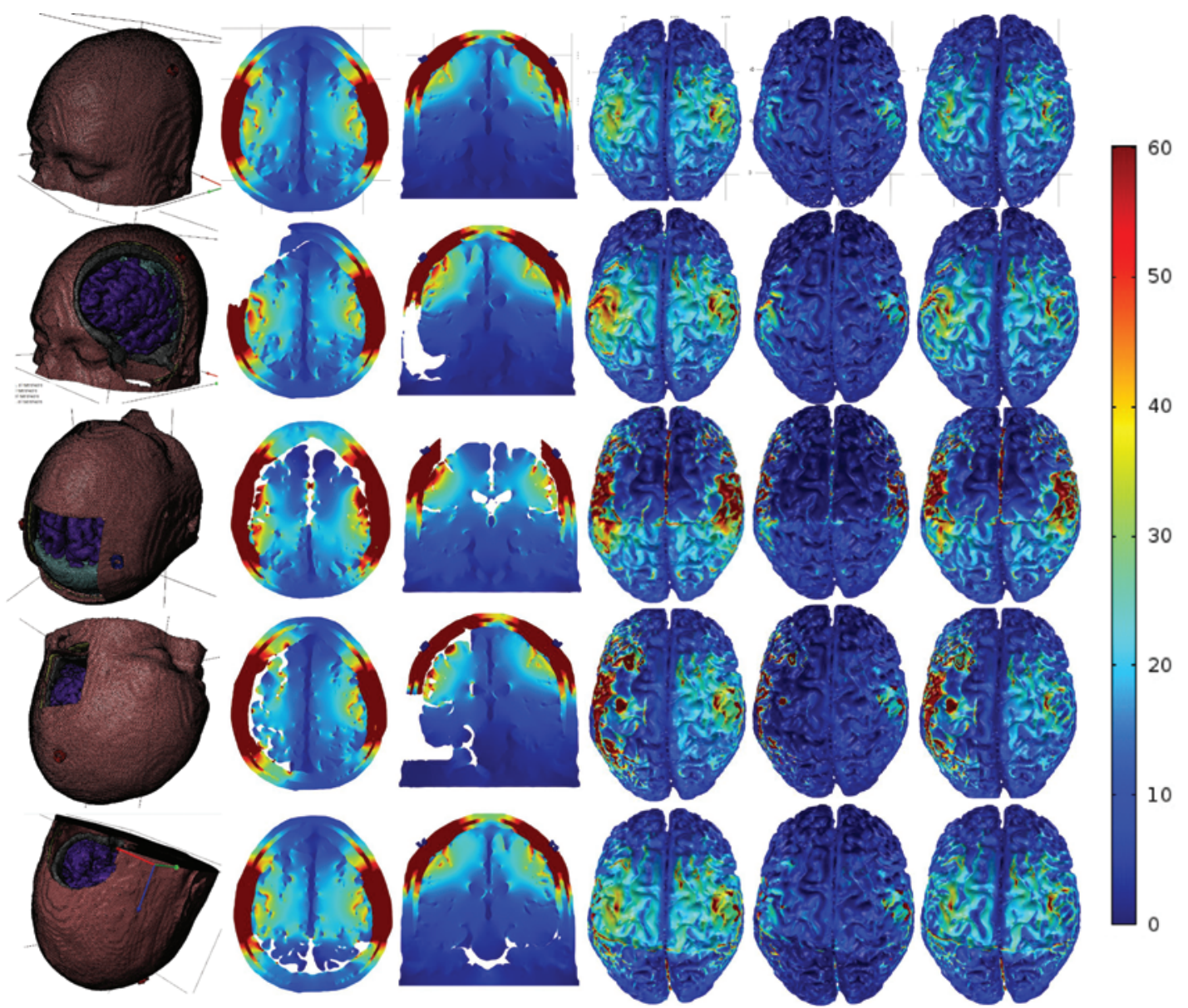

FIG. 4. The electric field of all CSF-decreased models is shown (from the top: normal head, frontotemporal craniotomy, parietal craniotomy, temporal craniotomy, and occipital craniotomy). Starting from the left, the 3D model view, axial section, coronal section, surface view of the electric field, pEF component, and tEF component are shown for each model. The color scale ranges from $0 \mathrm{~V} / \mathrm{m}$ (blue) to $60 \mathrm{~V} / \mathrm{m}(\mathrm{red})$. Figure is available in color online only.

CSF decrease from craniotomy was $26.6 \% \pm 45.6 \%$ on the craniotomy side and $7.34 \% \pm 14.0 \%$ on the unaffected side (independent sample t-test, $\mathrm{p}=0.112$ ) (Table 1). The percent increase after the CSF decrease was larger on the craniotomy side. The patient in Case 2, who underwent temporal craniotomy for the removal of a glioblastoma, had a UEtMEP threshold that was markedly increased after the CSF decrease (from $32 \mathrm{~mA}$ to $42 \mathrm{~mA}, 31.3 \%$ ). The brain sank following CSF drainage, and there was a space between the cortex and the skull (Fig. 7A). The patient in Case 5, who underwent trigeminal schwannoma removal via temporal craniotomy, had a UE-tMEP threshold on the craniotomy side that was decreased after the craniotomy (from $30 \mathrm{~mA}$ to $27 \mathrm{~mA},-10 \%$ ), but was then markedly increased following the CSF decrease (from $27 \mathrm{~mA}$ to $51 \mathrm{~mA}, 88.9 \%$ ) (Fig. 7C). The threshold of the unaffected side also increased $26.5 \%$, but the change was larger on the craniotomy side.

However, neither the patient in Case 3 nor the one in Case 4 showed a marked threshold increase during tumor removal. These patients had a petroclival meningioma removed via an anterior transpetrosal approach. Thus, the removal procedure was performed below the tentorium; the retractor lifted the brain epidurally from the subtemporal space and the frontal lobe was pressed against the convexity, so that there was no space between the motor cortex and the skull.

\section{Occipital Craniotomy and LSO Craniotomy}

We had only 1 occipital craniotomy case; surgery was performed with the patient in the prone position. This case showed no significant threshold change during surgery. On the other hand, we had 6 LSO case recordings. The head was rotated $45^{\circ}-60^{\circ}$ from the prone position and the vertex was tilted downward $30^{\circ}-45^{\circ}$. The differences in threshold changes between the craniotomy side and the other side after the craniotomy and the CSF decrease compared to the first recordings were small and not significant in these 6 LSO cases.

\section{Discussion}

\section{The 3D Model Simulation Study}

Several types of craniotomy and body positions are used in neurosurgery; however, the electrode location for tMEP monitoring is mostly at $\mathrm{C} 3-\mathrm{C} 4$, just above the hand motor area. We focused on the locational relationship of the craniotomy window and electrodes with body position during surgery. The electric field radially diffused from 
TABLE 1. Percent threshold change of the UE-tMEP during neurosurgery in 30 patients who underwent craniotomy

\begin{tabular}{|c|c|c|c|c|c|c|c|}
\hline \multirow{2}{*}{$\begin{array}{r}\text { Case No. } \\
\text { Frontote }\end{array}$} & \multirow{2}{*}{$\begin{array}{l}\text { Diagnosis } \\
\text { poral cran }\end{array}$} & \multicolumn{2}{|c|}{ 1st-2nd } & \multicolumn{2}{|c|}{ 1st-3rd } & \multicolumn{2}{|c|}{ 2nd-3rd } \\
\hline & & \multirow{2}{*}{$\frac{\text { Cran Side }}{\text { NA }}$} & \multirow{2}{*}{$\frac{\text { UA Side }}{\text { NA }}$} & \multirow{2}{*}{$\frac{\text { Cran Side }}{10}$} & \multirow{2}{*}{$\begin{array}{r}\text { UA Side } \\
6.25\end{array}$} & \multirow{2}{*}{$\frac{\text { Cran Side }}{\text { NA }}$} & \multirow{2}{*}{$\frac{\text { UA Side }}{\text { NA }}$} \\
\hline 1 & MCA aneurysm & & & & & & \\
\hline 2 & MCA aneurysm & 5.5 & 3.78 & 0.4 & 10.8 & -1.4 & 6.77 \\
\hline 3 & ICA aneurysm & -30.7 & 2.32 & -28.7 & -1.54 & 2.96 & -3.77 \\
\hline 4 & ACoA aneurysm & 3.22 & 4.27 & NA & NA & NA & NA \\
\hline 5 & MCA aneurysm & -5.7 & -15.1 & -27.3 & -20.2 & -22.8 & -5.96 \\
\hline 6 & High-grade glioma & -9.52 & NA & 4.54 & NA & 15.8 & NA \\
\hline 7 & Ependymoma & -2.47 & -2.78 & -16 & -2.86 & -13.9 & 0 \\
\hline 8 & TS meningioma & -20 & 0 & -11.4 & 6.25 & 10.7 & 6.25 \\
\hline 9 & Convexity meningioma & 27.8 & 7.14 & 16.9 & 0 & -5.8 & -4.5 \\
\hline 10 & Convexity meningioma & -38.8 & -7.69 & -34.7 & -7.69 & 6.67 & 0 \\
\hline 11 & ICA-AChA aneurysm & -17.8 & -3.57 & -10.7 & 7.14 & 8.7 & 10 \\
\hline 12 & ICA aneurysm & 3.7 & 5.1 & -3.7 & -5.1 & -6.9 & -9.76 \\
\hline 13 & MCA aneurysm & 3.7 & 2.7 & -10.8 & -2.7 & -13.1 & -5.4 \\
\hline \multicolumn{2}{|c|}{ Average \pm SD } & $-6.76 \pm 18.1$ & $-0.348 \pm 6.55$ & $-9.29 \pm 15.9$ & $-0.88 \pm 8.65$ & $-1.73 \pm 11.9$ & $-0.637 \pm 6.46$ \\
\hline \multicolumn{2}{|c|}{ Parietal cran (prone position) } & Rt Side & Lt Side & Rt Side & Lt Side & Rt Side & Lt Side \\
\hline 1 & Parasagittal meningioma & 68 & 37.9 & 92 & 37.9 & 14.3 & 0 \\
\hline 2 & Occipital AVM & -15.4 & -25.8 & -26.9 & -45.2 & -26.1 & -13.6 \\
\hline \multicolumn{2}{|c|}{ Bifrontal cran } & Rt Side & Lt Side & Rt Side & Lt Side & Rt Side & Lt Side \\
\hline 1 & Olfactory meningioma & 18.8 & 14.7 & 18.8 & 20.4 & 0 & 5.13 \\
\hline 2 & High-grade glioma & 0 & -4.16 & -8.33 & -4.16 & -8.33 & 0 \\
\hline 3 & Radiation necrosis & 17 & 18.9 & NA & NA & NA & NA \\
\hline \multicolumn{2}{|c|}{ Temporal cran } & Cran Side & UA Side & Cran Side & UA Side & Cran Side & UA Side \\
\hline 1 & Trigeminal neuralgia & NA & NA & 34 & 1.5 & NA & NA \\
\hline 2 & High-grade glioma & 7 & 16.7 & 40 & 8.3 & 31.3 & -7.1 \\
\hline 3 & Petroclival meningioma & 3.45 & 0 & 3.45 & 6.06 & 0 & 6.06 \\
\hline 4 & Petroclival meningioma & 11.5 & -1.9 & -3.8 & 1.9 & -13.8 & 3.9 \\
\hline 5 & Trigeminal schwannoma & -10 & 3 & 70 & 30.3 & 88.9 & 26.5 \\
\hline \multicolumn{2}{|c|}{ Average \pm SD } & $2.99 \pm 9.26$ & $4.45 \pm 8.41$ & $28.7 \pm 29.8$ & $9.61 \pm 11.9$ & $26.6 \pm 45.6$ & $7.34 \pm 14.0$ \\
\hline \multicolumn{2}{|c|}{ Occipital cran } & Rt Side & Lt Side & Rt Side & Lt Side & Rt Side & Lt Side \\
\hline 1 & Lower CN schwannoma & 7 & -3.7 & 10 & 1.86 & 3.1 & 5.77 \\
\hline \multicolumn{2}{|c|}{ LSO cran } & Cran Side & UA Side & Cran Side & UA Side & Cran Side & UA Side \\
\hline 1 & Lower CN schwannoma & 0 & 4.76 & 16.7 & -6 & -6 & 11.4 \\
\hline 2 & Vestibular schwannoma & NA & NA & 11.1 & 14.3 & NA & NA \\
\hline 3 & CPA epidermoid & -10.7 & -3.33 & -10.7 & 6.67 & 0 & 9.1 \\
\hline 4 & CPA meningioma & -6.25 & 2.86 & NA & NA & NA & NA \\
\hline 5 & CPA meningioma & -2.85 & -1.85 & -5.71 & -1.85 & -2.94 & 0 \\
\hline 6 & Vestibular schwannoma & -2.23 & 4.44 & 18.2 & 27.9 & 23.8 & 22.2 \\
\hline \multicolumn{2}{|c|}{ Average $\pm S D$} & $-4.41 \pm 4.17$ & $1.38 \pm 3.73$ & $5.92 \pm 13.3$ & $8.2 \pm 13.5$ & $3.72 \pm 13.6$ & $10.7 \pm 9.12$ \\
\hline
\end{tabular}

$\mathrm{AChA}=$ anterior choroidal artery; $\mathrm{ACoA}=$ anterior communicating artery; $\mathrm{CN}=$ cranial nerve; $\mathrm{CPA}=$ cerebellopontine angle; cran $=$ craniotomy; ICA $=$ internal carotid artery; $\mathrm{MCA}$ = middle cerebral artery; $\mathrm{NA}=$ not available; $\mathrm{TS}$ = tuberculum sellae; $\mathrm{UA}=$ unaffected; $1 \mathrm{st}$ = the first threshold recording before incision of the skin; 2nd = the second threshold recording after craniotomy; $3 r d=$ the third threshold recording after CSF drainage.

The percent change in threshold values is shown between the first recordings and postcraniotomy recordings as "1st-2nd"; between the first recordings and CSFdecreased recordings as "1st-3rd"; and between postcraniotomy recordings and the CSF-decreased recordings as "2nd-3rd" in each anodal stimulation side (defined as craniotomy side and unaffected side, or as left side and right side).

the brain surface at a maximum just below the electrodes; thus, the electric field was widely distributed between C3 and $\mathrm{C} 4$ electrodes in the frontal and parietal lobes. This distribution pattern is basic in the C3-C4 TES for tMEP monitoring, and craniotomy location and CSF decrease affect the distribution. ${ }^{10}$ 


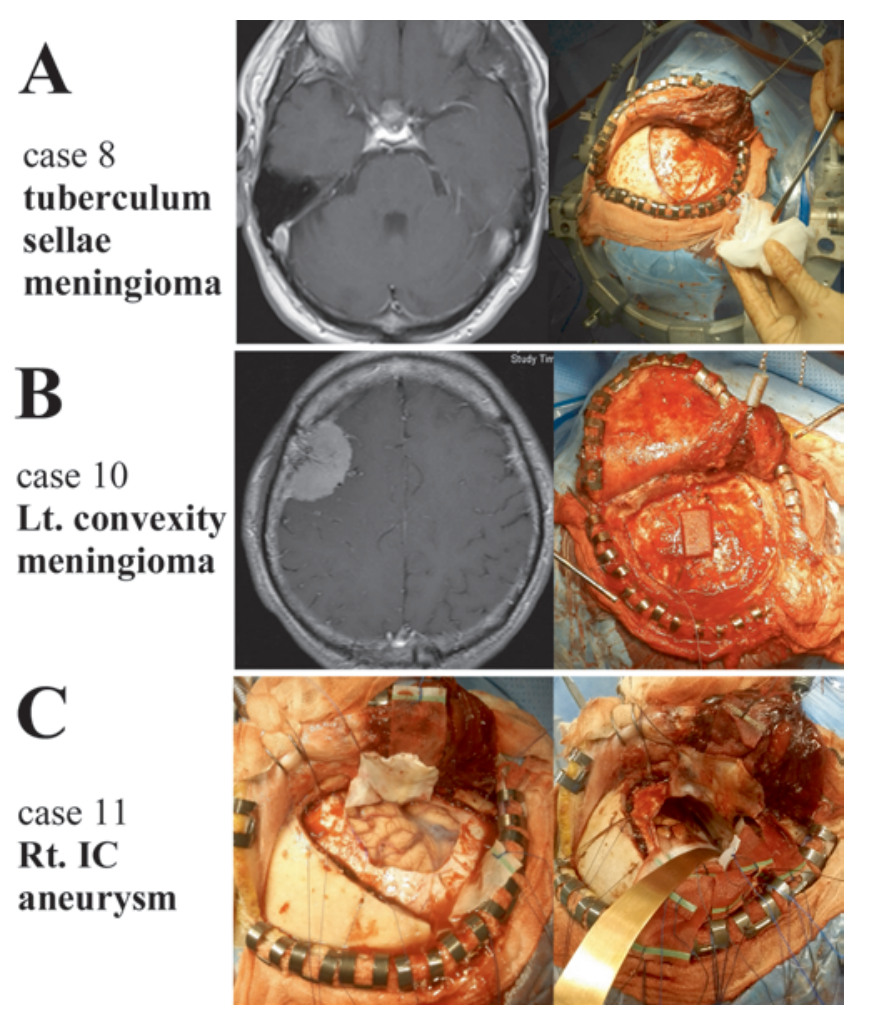

FIG. 5. Preoperative MR images and intraoperative photographs showing lesions and approaches in 3 frontotemporal craniotomy cases. A: Case 8. The patient had a tuberculum sellae meningioma removed through a right-side frontotemporal craniotomy. Cisternal CSF was drained, but the frontal lobe was lifted by the retractor after dural incision. B: Case 10. The patient had a left convexity meningioma removed. The craniotomy window was in close proximity to the motor cortex. The CSF was minimally drained after dural incision. C: Case 11. The patient had clipping of a right internal carotid artery (IC) aneurysm. Cisternal CSF was drained considerably, but the frontal lobe was also lifted by the retractor. Figure is available in color online only.

The bone layer removal causes a shift in the electric field distribution. It decreases the electric field in the brain under the craniotomy window, and increases under the bone at the margin of the craniotomy window. Thus, a higher electric field in the motor cortex was observed when the margin of the craniotomy window was near the motor cortex. This situation is possible in the frontotemporal, temporal, and parietal craniotomies. Our 3D models showed a slight increase in the electric field in the motor cortex after the bone removal in these craniotomy models. Although electric field gradient changes along the axon, which are called "activating functions," are considered to be more important than the magnitude of the electric field in a static state, the increase in the electric field in the motor cortex would be nearly equal to the increase in the efficacy of the TES. Thus, the threshold of the tMEP would decrease slightly after the bone removal in these types of craniotomy, and the effect of bone removal is smaller than that of the CSF removal.

The decrease in the CSF layer had a significant impact on the electric field in the brain in our simulation models. Air cannot conduct the electric current; therefore, there was no electric field in the area of the brain emerging
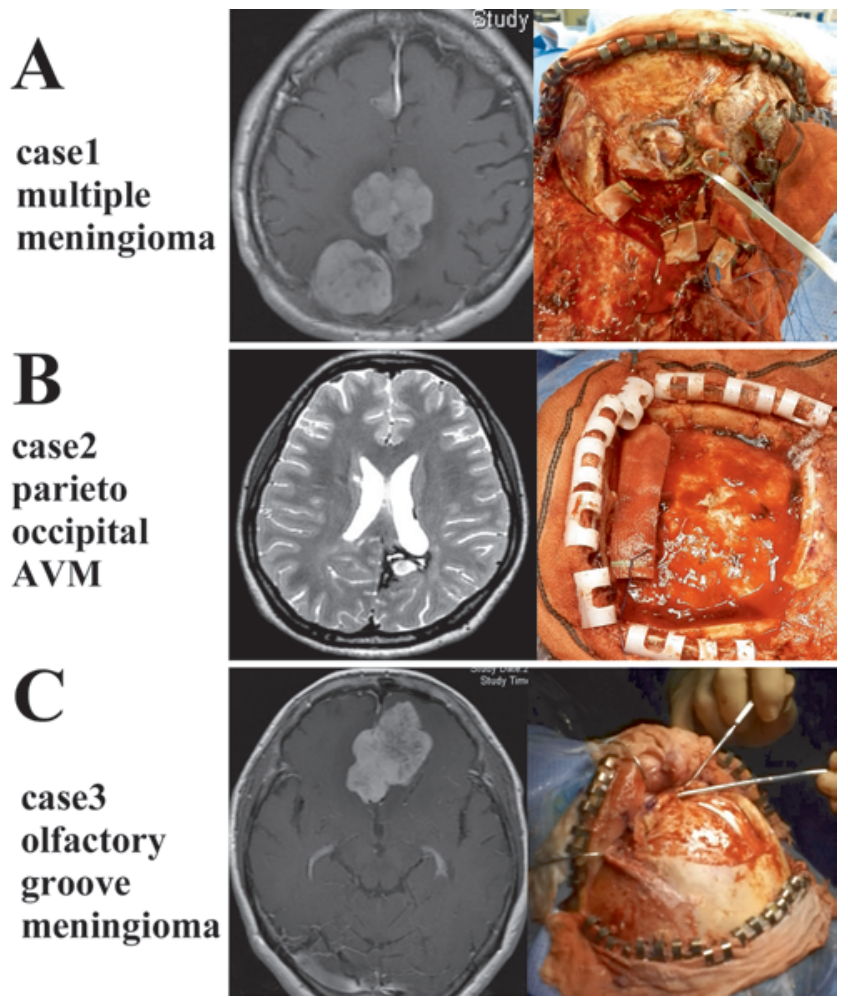

FIG. 6. Preoperative MR images and intraoperative photographs showing parietal and bifrontal craniotomy findings. A: Case 1 (parietal). The patient had multiple meningiomas removed via parietooccipital craniotomy in the prone position. The skin flap was widely inverted, and the electrodes were also detached from the skull. B: Case 2 (parietal). The patient underwent parietooccipital AVM removal in the prone position. The craniotomy window was located posterior-medial to the motor cortex and the frontal lobe sank, seeming to be pressed into the skull after dura incision. C: Case 3 (i.e., Case 1 for bifrontal). The patient had an olfactory groove meningioma removed via bifrontal craniotomy, and the craniotomy window was more anterior than the motor cortex. Figure is available in color online only.

from the CSF surface. Meanwhile, extremely high electric fields were confirmed at points where the brain had direct contact with the skull above the CSF. In addition, high-value areas in the brain were observed just under the CSF surface line. The exposure of the brain following CSF decrease creates the uneven distribution and concentration of the electric field. This deviation of the electric field caused by a large CSF decrease would cause unexpected changes in tMEP monitoring. The tMEP threshold should increase when the motor cortex is exposed, due to the CSF decrease and the brain not being attached to the skull, but when the motor cortex is located just under the CSF surface or if there is direct contact with the skull, the threshold should decrease. Sometimes, the motor cortex is exposed to air during a long-duration craniotomy, especially in patients with an atrophic brain.

The parietal craniotomy is the most affected by the CSF decrease in our simulation model, because the motor cortex can easily be exposed to air due to the craniotomy location and body position. The occipital craniotomy appears to be minimally affected by the CSF decrease in our model because the CSF layer over the motor cortex usually 


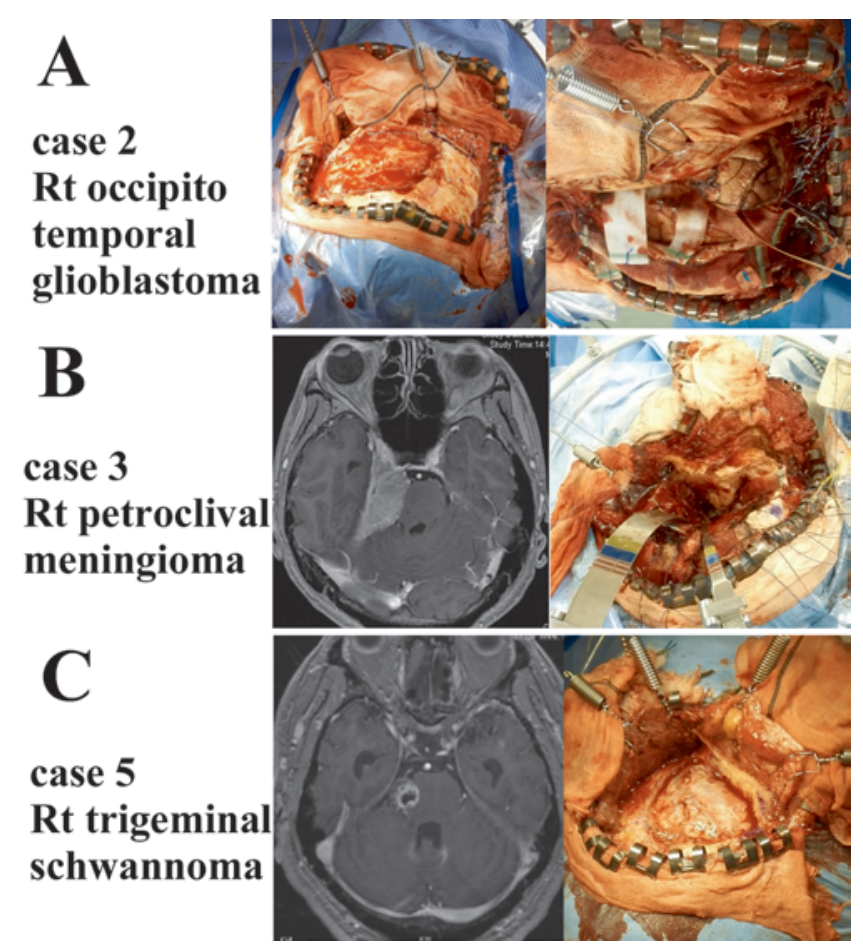

FIG. 7. Preoperative MR images and intraoperative photographs showing temporal craniotomy findings. A: Case 2. The patient underwent right occipitotemporal glioblastoma removal. It was obvious that the brain sank after dural incision. B: Case 3. The patient had a right petroclival meningioma removed. The brain was lifted using the retractor epidurally away from the subcentral space. C: Case 5 . The patient had a right trigeminal schwannoma removed also via temporal craniotomy. The brain sank after dural incision and was supported only by the retractor. Figure is available in color online only.

remains intact during this type of craniotomy in the prone position.

\section{Clinical Validation}

The 3D model bone removal findings were consistent with our limited clinical experience of the tMEP. In fact, the tMEP thresholds in our clinical cases were decreased in the frontotemporal craniotomy. This threshold change was significantly larger on the craniotomy side than on the unaffected side. Although we did not have enough cases to validate the electric field change after the temporal and parietal craniotomies, the threshold decrease was reasonable when the motor cortex was located near the craniotomy window, as in our frontotemporal craniotomy cases.

The UE-tMEP threshold recordings were markedly increased after the CSF decrease in 3 of 5 temporal craniotomy cases, except in the 2 cases of petroclival meningioma removal via an anterior transpetrosal approach in which the retractor lifted the brain epidurally from the subtemporal space. The motor cortex is prone to being exposed to the air during standard temporal craniotomies due to the head position, and the distance between the motor cortex and the skull is also prone to expand due to the effects of gravity and the decrease in CSF that cause the brain to shift. Thus, it was reasonable that the threshold increased after the CSF decrease during the temporal craniotomy.
On the other hand, the threshold change was small after the CSF decrease in our frontotemporal craniotomy cases. The primary motor cortex was not exposed, and the frontal lobe was lifted against the convexity by a retractor during the pterional approach in most cases. Therefore, the CSF layer over the motor cortex was retained and the distance between the motor cortex and the skull was short, so the threshold changes were small. Unfortunately, we did not have a similar clinical case for our parietal craniotomy model. We had only 2 parietal craniotomy cases treated with patients in a prone position, and the situation of both cases was considerably different than in our simulation model.

Although our clinical occipital craniotomy cases included only 1 midline suboccipital craniotomy and 6 LSO craniotomies, there was no tendency for the threshold to change after both the craniotomy and the CSF decrease. The occipital craniotomy is considered to minimally affect the threshold after bone removal because the bone window is far from the motor cortex.

Goto et al. also reported that the threshold change from the initial recording before the skin incision to the final recording after the operation was significantly larger on the craniotomy side $(5.5 \%, \mathrm{n}=51)$ than on the unaffected side $(1.1 \%, n=40)(p=0.038)$ (Goto $T$ et al: Motor threshold stimulation in intraoperative motor evoked potential monitoring, presented at the 25 th Conference on Neurosurgical Techniques and Tools, Niigata, Japan, 2016). They estimated the tMEP threshold change in patients without any pre- or postoperative motor weakness. Most of their recording cases were from supratentorial surgeries, with only 6 infratentorial surgery cases that had recordings from both sides. Their finding was also consistent with our results that the threshold of the craniotomy side was prone to be affected by surgical procedures.

Some of the clinical recording results showed that our FE models could accurately simulate the electric field change in the brain after a craniotomy. However, the CSF decrease pattern appeared to be more limited or complicated in real neurosurgical situations, so our CSF-decreased model may be an oversimplification. When a compartment of the CSF layer over the motor cortex does not diminish or the motor cortex has direct contact with the skull, there should be no negative effect in the generation of the electric field in the brain. The recordings of the frontotemporal craniotomy cases showed no significant threshold change after the CSF decrease on the craniotomy side. The dramatic changes of the electric field in the brain after CSF decrease only occur when there is air between the motor cortex and the skull. Such a large increase in the threshold after CSF decrease in a real setting was recorded only in some temporal craniotomy cases.

Another problem with our FE model simulation is the brain shift. There is always a brain shift during subdural procedures in neurosurgery because the brain sinks when CSF decreases, but the brain can be raised by a retractor. Thus, the distance between the motor cortex and the skull depends on the degree of brain shift. Our present models cannot account for this brain shift. Theoretically, the electric field in the brain decreases if the distance shortens and increases if the distance lengthens. 
Our speculation from this simulation model may be validated by some of our clinical recordings, but more clinical recordings are needed to estimate the effects of the changes that occur during these craniotomies. A 3D model that can simulate the brain shift should be developed to perform a more accurate estimation of the electric field change from these craniotomies.

Nevertheless, this study clearly demonstrates that the tMEP threshold is expected to fluctuate depending on the craniotomy surgery. Major CSF decreases and brain shifts would negatively affect tMEP ignition. These changes should be minimized to maintain the original conductivity between the motor cortex and the skull. However, a certain level of change is unavoidable during craniotomy surgeries. Thus, the operating team must keep in mind the threshold fluctuation during the craniotomy, and obtain an intraoperative baseline just before beginning the main portion of the procedure to estimate genuine changes in motor function.

\section{Conclusions}

Bone removal during a craniotomy positively affects the generation of the electric field in the motor cortex if the motor cortex is just under the bone at the margin of the craniotomy window. A major decrease in CSF during an operation has a significantly negative impact on the electric field when the motor cortex is exposed to air. The CSF decrease and brain shift can negatively affect tMEP ignition. These changes should be minimized to maintain the original conductivity between the motor cortex and the skull, and the operating team must remember the fluctuation of the tMEP threshold.

\section{Acknowledgments}

This work was supported by the Keio University Doctorate Student Grant-in-Aid Program. We thank Hashiguchi (Keisoku Engineering, Tokyo) for his contributions to technical support of Comsol Multiphysics.

\section{References}

1. Holdefer RN, Sadleir R, Russell MJ: Predicted current densities in the brain during transcranial electrical stimulation. Clin Neurophysiol 117:1388-1397, 2006

2. Lee JJ, Kim YI, Hong JT, Sung JH, Lee SW, Yang SH: Intraoperative monitoring of motor-evoked potentials for supratentorial tumor surgery. J Korean Neurosurg Soc 56:98-102, 2014

3. Miranda PC, Mekonnen A, Salvador R, Ruffini G: The electric field in the cortex during transcranial current stimulation. Neuroimage 70:48-58, 2013
4. Motoyama Y, Kawaguchi M, Yamada S, Nakagawa I, Nishimura F, Hironaka Y, et al: Evaluation of combined use of transcranial and direct cortical motor evoked potential monitoring during unruptured aneurysm surgery. Neurol Med Chir (Tokyo) 51:15-22, 2011

5. Rampersad SM, Stegeman DF, Oostendorp TF: Single-layer skull approximations perform well in transcranial direct current stimulation modeling. IEEE Trans Neural Syst Rehabil Eng 21:346-353, 2013

6. Szelényi A, Bueno de Camargo A, Flamm E, Deletis V: Neurophysiological criteria for intraoperative prediction of pure motor hemiplegia during aneurysm surgery. Case report. J Neurosurg 99:575-578, 2003

7. Szelényi A, Hattingen E, Weidauer S, Seifert V, Ziemann U: Intraoperative motor evoked potential alteration in intracranial tumor surgery and its relation to signal alteration in postoperative magnetic resonance imaging. Neurosurgery 67:302-313, 2010

8. Szelényi A, Langer D, Kothbauer K, De Camargo AB, Flamm ES, Deletis V: Monitoring of muscle motor evoked potentials during cerebral aneurysm surgery: intraoperative changes and postoperative outcome. J Neurosurg 105:675681,2006

9. Tanaka S, Tashiro T, Gomi A, Takanashi J, Ujiie H: Sensitivity and specificity in transcranial motor-evoked potential monitoring during neurosurgical operations. Surg Neurol Int 2:111, 2011

10. Tomio R, Akiyama T, Horikoshi T, Ohira T, Yoshida K: Visualization of the electric field evoked by transcranial electric stimulation during a craniotomy using the finite element method. J Neurosci Methods 256:157-167, 2015

11. Zhou HH, Kelly PJ: Transcranial electrical motor evoked potential monitoring for brain tumor resection. Neurosurgery 48:1075-1081, 2001

\section{Disclosures}

The authors report no conflict of interest concerning the materials or methods used in this study or the findings specified in this paper.

\section{Author Contributions}

Conception and design: Tomio. Acquisition of data: Tomio. Analysis and interpretation of data: Tomio. Drafting the article: Tomio. Critically revising the article: Tomio. Reviewed submitted version of manuscript: all authors. Approved the final version of the manuscript on behalf of all authors: Tomio. Administrative/ technical/material support: Tomio. Study supervision: Tomio, Akiyama.

\section{Correspondence}

Ryosuke Tomio, Department of Neurosurgery, Keio University School of Medicine, 35 Shinanomachi, Shinjuku, Tokyo 1608582, Japan. email: tomy0807@ hotmail.com. 